\title{
Your DNA is not your diagnosis: getting diagnoses right following secondary genomic findings
}

\author{
Michael F. Murray, MD¹
}

The recent report of a pathogenic variant of one of the American College of Medical Genetics and Genomics's 56 genes in a supercentenarian (i.e., a person 110 years or older) without overt disease highlights the significant gaps in our understanding of penetrance related to secondary genomic findings. ${ }^{1}$ There are a growing number of venues in which incidental or secondary genomic findings are returned to patients. Secondary findings are being returned in clinical care settings as well as research settings., ${ }^{2,3}$ As the number of patients affected by these results rises, so too will the number of people in whom a correlation between genotype and phenotype is not observed.

All medical diagnoses are achieved through a process. ${ }^{4}$ The diagnostic process typically starts with a symptom, a complaint, or a clinical finding. The health-care system is then engaged to generate diagnostic hypotheses to explain the finding, test those hypotheses, and then accept or reject those hypotheses. Ultimately, all of the pertinent positives and pertinent negatives need to be assembled under an acceptable working diagnosis. In approaches that generate "primary" genomic findings, DNAbased testing is typically applied late in the diagnostic process. ${ }^{5}$ What has been referred to as "diagnostic" genomic sequencing is employed with the intent of confirming the clinical suspicion that a genotype-phenotype correlation can be identified to explain an extensively studied phenotype. ${ }^{5}$ However, in the setting of incidental or secondary findings, the DNA-based testing prompts the initiation of a diagnostic process that must then be followed up by an appropriate clinical evaluation.

The concepts of pretest probability as well as positive predictive value (PPV) are important to consider as they relate to this issue. Detailed discussions of these and related terms are widely available; $^{6}$ for this discussion, however, pretest probability is best understood as the likelihood that the genomic condition in question is present before the secondary finding is uncovered in the patient, and the PPV of the test is the number of truepositive results generated divided by the number of all positive results. By definition, incidental and secondary findings are "the results of a deliberate search for pathogenic or likely pathogenic alterations in genes that are not apparently relevant to a diagnostic indication for which the sequencing test was ordered," and therefore they are not linked to the test indication (be it clinical or research-based testing). ${ }^{7}$ In the case of secondary genomic findings, the pretest probability is primarily linked to the prevalence of the condition among the relevant population. It is important to note that even for the most highly sensitive and specific tests, a low pretest probability for a condition correlates with a low PPV for the testing ${ }^{6}$ (Table 1 ).

In the arena of secondary findings, the PPV of the testing process that generates the secondary finding is expected to be low, and this test result alone never constitutes a diagnosis. Once identified, each individual case requires additional data to either move patients along the diagnostic process or to reject the diagnostic hypothesis. Data related to family history, personal history, physical examination, further diagnostic testing, and interpretation should be pursued in each case. ${ }^{8}$ This secondary finding scenario is, of course, very different from the scenario where a specific gene is sequenced as part of a quest to make a specific genotype-phenotype correlation; in the setting of targeted genetic testing, the pretest probability for the condition is often greater than $50 \%$ and the PPV of the testing result is often over $90 \%^{6}$ (Table 1 ).

Until data-driven pretest probabilities and PPVs for secondary genomic findings are understood in larger populations, it is reasonable to anticipate that "genotype without phenotype" will occur at frequencies that are greater than or equal to such instances of nonpenetrance when targeted genetic testing for a condition is pursued. The experience of targeted genetic testing for hemochromatosis versus population-based genetic screening for hemochromatosis has led to an appreciation of the potential for significantly larger percentages of "genotype without phenotype" to be found in populations with a lower pretest probability. ${ }^{9}$

Figure 1 displays a model for classifying possible outcomes of a diagnostic workup triggered by an incidental or secondary finding. In groups 1,2 , and 3 , there is a genotype-phenotype correlation, and a resultant genomic syndrome diagnosis is achieved. In groups 4 and 5, there is genotype without phenotype, and there is no genomic syndrome diagnosis. The patients in whom the phenotype is absent (i.e., groups 4 and 5) need to be followed clinically over time to determine whether a phenotype will emerge or if a genomic mechanism for nonpenetrance

${ }^{1}$ Clinical Genomics, Geisinger Health System, Forty Fort, Pennsylvania, USA. Correspondence: Michael F. Murray (mfmurray1@geisinger.edu)

Submitted 1 August 2015; accepted 31 August 2015; advance online publication 8 October 2015. doi:10.1038/gim.2015.134 
can be found. Condition-specific risk-management strategies to be applied to those in whom groups 4 and 5 cannot be distinguished need to be formulated. The potential harm of incorrectly labeling patients with the diagnosis of a genetic condition who ultimately end up in group 5 should not be underestimated; such inappropriate diagnosis could unfairly limit insurability or other important activities.

Secondary genomic findings should be viewed as biomarkers. They can be used to highlight possible disease risk, but in some cases such findings may never be associated with penetrant disease in the patients who carry them. Just as C-reactive protein

Table 1 The relationship between the pretest probability of disease and both the positive and the negative predictive values associated with an idealized laboratory test in which there is a $95 \%$ sensitivity and a $95 \%$ specificity

\begin{tabular}{lccccc} 
Predictive & \multicolumn{5}{c}{ Pretest probability of disease } \\
\cline { 2 - 6 } $\begin{array}{l}\text { value of the } \\
\text { test }\end{array}$ & $\mathbf{9 9 \%}$ & $\mathbf{8 0} \%$ & $\mathbf{5 0 \%}$ & $\mathbf{2 0 \%}$ & $\mathbf{1 \%}$ \\
\hline Positive & 99.9 & 99 & 95 & 83 & 16 \\
\hline Negative & 16 & 83 & 95 & 99 & 99.9 \\
\hline
\end{tabular}

Note that the sensitivity and specificity of calling secondary genomic variants has not been formally studied and likely will be specific to the condition; the pretest probability will be specific to the population.

Adapted from ref. 6 . is a biomarker for coronary artery disease risk ${ }^{10}$ but does not stand as a proxy for a diagnosis of coronary artery disease, so too are secondary genomic variants not diagnoses. Most electronic health records allow for problem lists in which patients in groups 4 and 5 can have their genomic variant listed for consideration by providers until new data can be added to move it to a diagnosis or remove it from the problem list.

As patients are increasingly informed of secondary genomic findings and placed in the challenging situation of a "genotype without phenotype," the health systems that care for them will require new policies and management strategies. Population screening with genomic sequencing will extend this phenomenon to larger groups of individuals.

The professional genetics community should take the lead in making clear to patients and families, other providers, the medical community at large, and payers that there are important distinctions between an isolated secondary genomic finding and a genomic diagnosis. Distinctions must be made in order to avoid inappropriately grouping individuals who have a "genetic risk" with individuals who have a "genetic diagnosis." The DNA finding together with the clinical findings should guide management, follow-up, and the label.

\section{DISCLOSURE}

The author declares no conflict of interest.

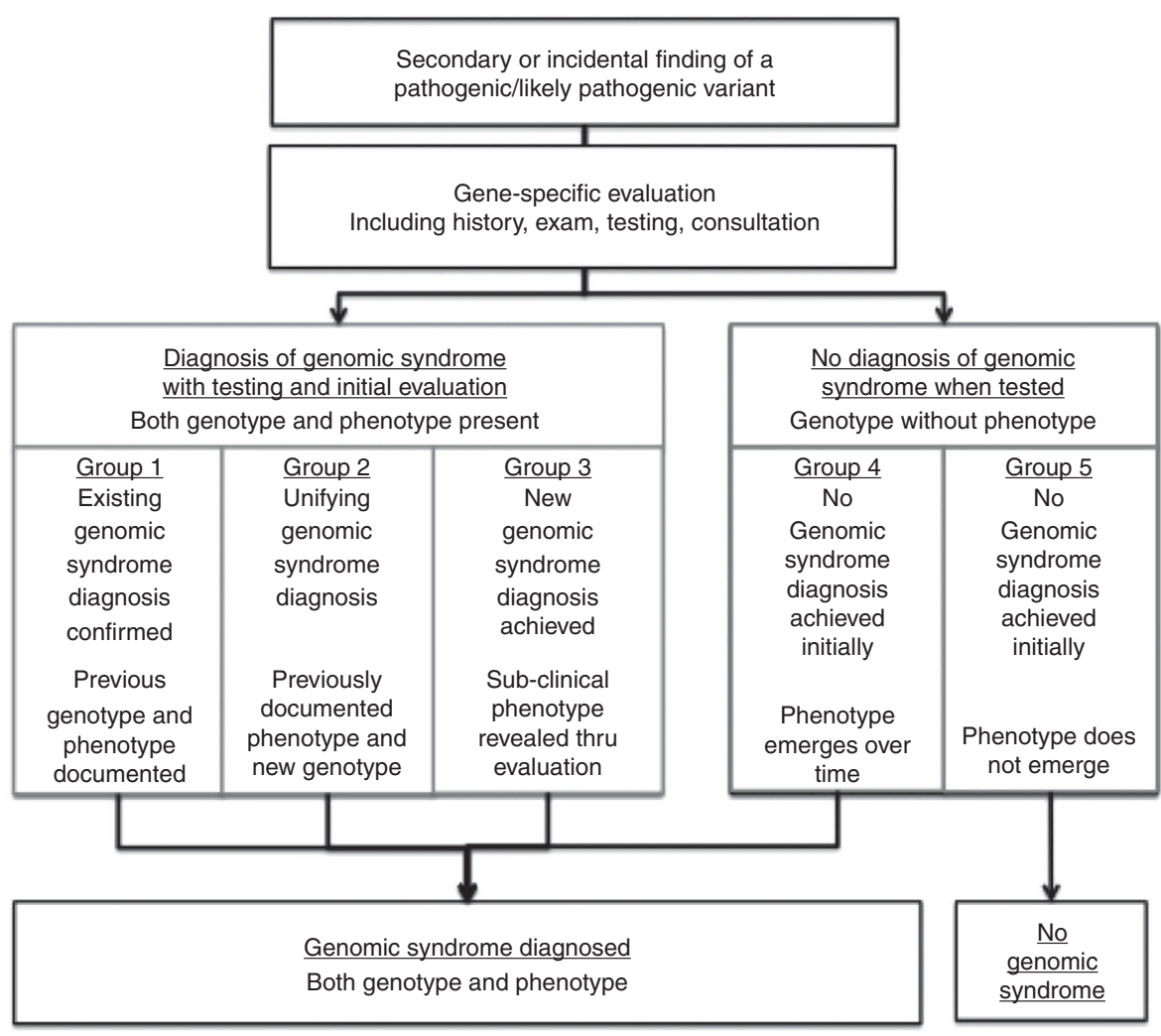

Figure 1 A model for the classification of patients with incidental or secondary findings on genomic sequencing. Current strategies for genotype-phenotype correlation cannot distinguish group 4 from group 5. The increasing identification of patients who fit into "genotype without phenotype" (i.e., groups 4 and 5) creates a need for new management plans and health-care policies. 


\section{REFERENCES}

1. Gierman HJ, Fortney K, Roach JC, et al. Whole-genome sequencing of the world's oldest people. PLoS One 2014;9:e112430.

2. Yang $Y$, Muzny DM, Xia F, et al. Molecular findings among patients referred for clinical whole-exome sequencing. JAMA 2014;312:1870-1879.

3. Berg JS, Amendola LM, Eng C, et al.; Members of the CSER Actionability and Return of Results Working Group. Processes and preliminary outputs for identification of actionable genes as incidental findings in genomic sequence data in the Clinical Sequencing Exploratory Research Consortium. Genet Med 2013;15:860-867.

4. Baerheim A. The diagnostic process in general practice: has it a two-phase structure? Fam Pract 2001;18:243-245.

5. Biesecker LG, Green RC. Diagnostic clinical genome and exome sequencing. NEngl J Med 2014;371:1170.
6. Scherokman B. Selecting and interpreting diagnostic tests. Perm J 1997;1:4-7. http://www.thepermanentejournal.org/issues/1997/fall.html. Accessed 31 May 2015.

7. Green RC, Berg JS, Grody WW, et al.; American College of Medical Genetics and Genomics. ACMG recommendations for reporting of incidental findings in clinical exome and genome sequencing. Genet Med 2013;15: 565-574.

8. Kohane IS, Hsing M, Kong SW. Taxonomizing, sizing, and overcoming the incidentalome. Genet Med 2012;14:399-404.

9. Alexander J, Kowdley KV. HFE-associated hereditary hemochromatosis. Genet Med 2009;11:307-313.

10. Tsimikas S, Willerson JT, Ridker PM. C-reactive protein and other emerging blood biomarkers to optimize risk stratification of vulnerable patients. J Am Coll Cardio/ 2006;47(8 Suppl):C19-31. 